\title{
Develop an Intelligence Analysis Tool for Abdominal Aortic Aneurysm
}

\author{
Nan-Chen Hsieh, Jui-Fa Chen, and Hsin-Che Tsai
}

\begin{abstract}
An Abdominal Aortic Aneurysm (AAA) is a focal dilatation at some point of the abdominal section of the aorta. In the absence of any treatment, AAA tends to grow until rupture. In this paper, we develop an Intelligence Analysis Tool to help researchers predict postoperative morbidity after AAA. The Tool includes an ensemble model, classification modules, model evaluation, and data visualization. The probabilities of complication calculated by the model of complications and a receiver operating characteristic (ROC) curve were used to evaluate the accuracy of postoperative morbidity prediction. The results show that the system proposed by this approach yields valuable qualitative and quantitative information for postoperative morbidity of Abdominal Aortic Aneurysm patients. Our System facilitates different types of users to access without to learn new data mining software.
\end{abstract}

\section{Introduction}

Cardiac surgery is a complex surgical operation that is indicated for patients with severe insufficiency in cardiac function. Major cardiac surgical interventions include coronary artery bypass grafting $(\mathrm{CABG})$, repair of congenital heart defects, surgical treatment of atrial fibrillation, heart transplantation, repair or replacement of heart valves, aortic surgery, aneurysm repair or a combination of

\footnotetext{
Nan-Chen Hsieh

Department of Information Management, National Taipei University of Nursing and Health Sciences, Taiwan, ROC

e-mail: nchsieh@gmail.com

Jui-Fa Chen $\cdot$ Hsin-Che Tsai

Department of Computer Science and Information Engineering, Tamkang University, Taiwan, ROC

e-mail: alpha@mail.tku.edu.tw, shin@live.com
} 
these surgical procedures. During the operation and the postoperative stay at the Intensive Care Unit (ICU) and nursing ward, there is considerable morbidity for cardiac surgery patients with postoperative complications, which results in increased hospital mortality and postoperative morbidity. Many prediction models for cardiac surgical outcome apply logistic or multivariable regression to assess preoperative risk [1-3]. An Abdominal Aortic Aneurysm in the absence of any treatment, tend to grow until rupture. There are two main techniques for Abdominal Aortic Aneurysm reparation: Open Repair (OR), which is an invasive surgical procedure, and the Endovascular Aneurysm Repair (EVAR), which is a minimally invasive procedure where a stent graft is inserted using a catheter in order to exclude the bulge from the blood circulation. Most risk assessment tools used traditional statistical method to derive prediction models for patients who were undergoing open abdominal aortic aneurysm repair, and to evaluate whether or not the surgical procedures would be successful. Similarly, postoperative morbidity is a key factor in recovery and through-put of cardiac hospital patients. Prediction of surgical mortality and postoperative morbidity is important in selecting low-risk patients for operation, and in counseling patients about the risks of undergoing surgical operation. The development of a robust prediction model can therefore both assist vascular surgeons in evaluating the expected outcome for a given patient and facilitate counseling and preoperative decision-making. Reliable and accurate prediction of operative mortality and morbidity is an essential criterion for any such risk evaluation models.

Data mining techniques are currently used in medical decision support to increase diagnostic accuracy and to provide additional knowledge to medical stuff. Their increased use provides expanded opportunities for determining the utility of medical decision making models from retrospective data. Prediction and description are two distinct concepts in data mining. For medical application, data mining not only support prediction models with satisfactory accuracy, but also needs a safety context in which decision-making activities require more explanatory support. The main distinction is that predictive data mining requires that the training dataset include an outcome variable, while descriptive data mining uses a global strategy to find the characteristics of each affinity granulation of the data. Both these data mining techniques can produce accurate, predictive and interpretable descriptive models that contribute greatly to handle medical data gathered through systematic use of clinical, laboratory, and hospital information systems. The goal of predictive data mining in clinical surgery is to derive models that can use medical data to predict patient's mortality and morbidity and thereby support clinical surgical decision-making. Predictive data mining can also aid in prognosis, diagnosis and treatment planning for surgical procedures. In contrast, descriptive data mining considers the data as affinity granulations, and aims at finding interpretable patterns and associations among data.

The use of machine learning models has become widely accepted in medical applications. Delen et al [4] developed a Web-based Decision Support Systems, which are used to build different types of prediction models (including neural networks, decision trees, ordinal logistic regression and discriminant analysis) to classify a movie. Murphy [5] presented a Clinical Decision Support System use of 
Decision Tree is one of the most popularly applied methods. Artificial Neural Network (ANN) has featured in a wide range of medical applications, often with promising results. Eom et al. [6] developed a classifier ensemble-based, including Artificial Neural Network (ANN), Decision Tree (DT), and Support Vector Machine (SVM), clinical decision support system for predicting cardiovascular disease level. SVM have been successfully used in a wide variety of medical applications. Polat and Güne [7] used a least square support vector machine to assist breast cancer diagnosis. Babaoghlu et al. [8] first used principle component analysis method to reduce data features, and acquired an optimum support vector machine model for the diagnosis of coronary artery disease. Choi [9] proposed the detection of valvular heart disorder (VHD) by wavelet packet decomposition and SVM techniques.

This study describes the development of an informative ensemble prediction model in web-based system, consisting of DT, ANN and SVM for the prediction of postoperative morbidity between preoperative variables and complication outcomes in Abdominal Aortic Aneurysm patients. For a better understanding of our study, Section 2 of this paper begins with an overview of study background and system overview in general. Section 3 describes the procedures used in this study, ensemble model for the prediction of postoperative morbidity. Section 4 discusses the experimental findings and offers observations about practical applications and directions for future research.

\section{System Overview}

Hospital mortality is an important clinical endpoint in major Abdominal Aortic Aneurysm. A prognostic model is often used for clinical decision-making prior to and during such surgery. The predicted outcome can be used by surgeons and patients to evaluate whether or not the surgical procedures is likely to be successful. Similarly, postoperative morbidity is a key factor in recovery and throughput of cardiac hospital patients. Prediction of surgical mortality and postoperative morbidity is important in selecting low-risk patients for operation, and in counseling patients about the risks of undergoing surgical operation. The development of a robust prediction model can therefore both assist vascular surgeons in evaluating the expected outcome for a given patient and facilitate counseling and preoperative decision-making. Reliable and accurate prediction of operative mortality and morbidity is an essential criterion for any such risk evaluation models.

The Weka workbench contains a collection of visualization tools and algorithms for data analysis and predictive modeling, together with graphical user interfaces for easy access to this functionality. It is freely available software. It is portable and platform independent because it is fully implemented in the Java programming language and thus runs on almost any modern computing platform. Weka has several standard data mining tasks, data preprocessing, clustering, classification, association, visualization, and feature selection[10]. But it is not easily accessible and inconvenient to use data analysis functions. We developed Intelligence Analysis Tool as a web application, the tool can be installed and used by 
any client in the network. The corresponding algorithms in Weka library enabled data analysis tasks by interaction with the web service, and returned the analyzed results. Figure 1 shows the tool's architecture.

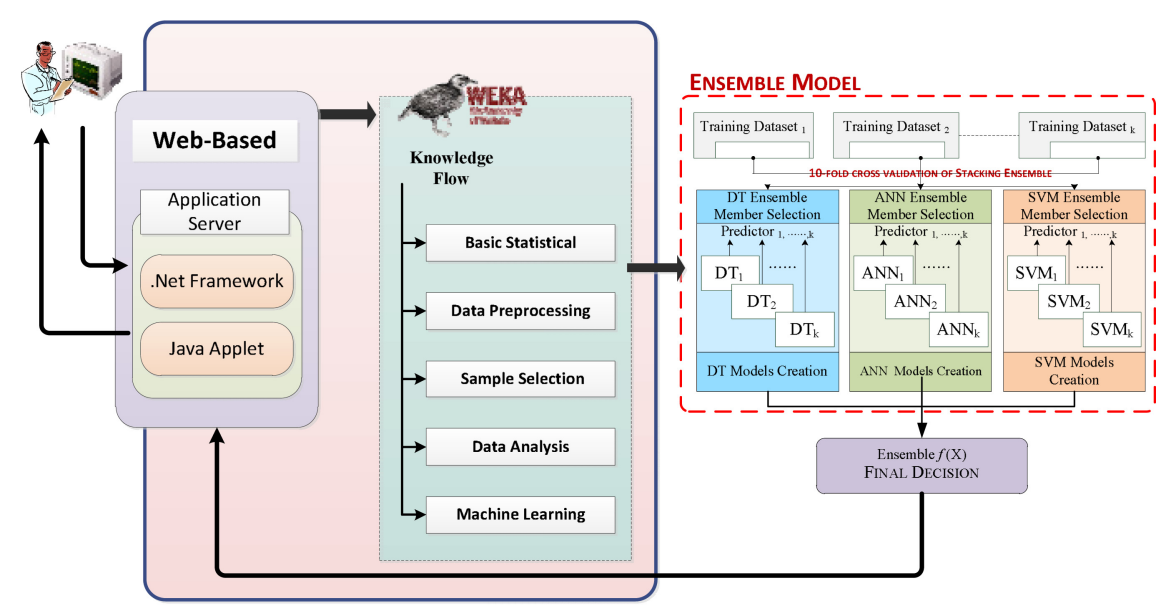

Fig. 1 The proposed architecture

The Intelligent Analysis Tool descriptive statistics functions and Data Mining functions were supported by Weka library. The Intelligent Analysis Tool descriptive statistics functions and Data Mining functions were supported by Weka library. User can choose ensemble model including DT, ANN and SVM to construct a set of hypotheses to predict risk. This study designed intelligent Analysis Tool facilitates different types of users to access functionalities in Weka without the need to learn new data mining software.

\section{Method and Procedures}

Abdominal Aortic Aneurysm is an enlargement that occurs in a weakened area within the largest artery in the abdomen (http://www.vascularweb.org/patients/ NorthPoint/Abdominal_Aortic_Aneurysm.html). If an AAA is not treated in due time, the pressure generated by heartbeats causes the aneurysm to continuously grow larger, and the aortic wall continues to weaken. Finally, a rupture occurs that causes massive internal bleeding. The best way to prevent the high mortality associated with AAA is to find the lesion before rupture occurs. However, patients with aortic diseases are often elderly with severe co-morbidities and sometimes devastating morbidity, making them extremely challenging candidates for surgery. 


\subsection{Input Data}

We retrospectively examined 122 consecutive patients who underwent Open Repair surgery at Taipei Veteran General Hospital, a teaching hospital in Taiwan, between 2007 and 2009. The dataset contains preoperative patient characteristics, details of the operative information, and pathological and laboratory findings from the emergency room, operating room and intensive care unit. The dataset also included length of ICU stay, variables that describe postoperative complications that frequently occur in Open Repair surgery, death during hospitalization, and time of death for patients who expired. Postoperative complication was used as the binary outcome variable of the ensemble model, and types of complications were used as subsidiary outcome variables. The original dataset contained 154 variables, but included many missing values. Preliminary inspection of the dataset showed that many variables contained missing values for at least $50 \%$ of the patients; these variables were not included in further analysis. In order to identify significant variables for use in the ensemble model, a number of criteria were employed. Variables that were subjective, ambiguous or inadequately defined were excluded; variables that were frequently incomplete were also excluded from subsequent analysis. Data collected included preoperative patient characteristics, risk factors, details of the operative information, physical characteristics of the aneurysm, postoperative physiological and laboratory findings, and postoperative complications as the outcome variable.

\subsection{Ensemble Model for Prediction}

DTs, ANNs, and SVMs were chosen as basis models for the ensemble model, because they represented different approaches, each of which is simple to implement and has been shown to perform well in medical applications. The rationale of employing these models is that Decision Tree have simplicity and capacity for humanly understandable inductive rules so many researchers use Decision Tree to resolve problems and error analysis. Artificial Neural Network is generally superior to conventional statistical models, and Support Vector Machine performs reasonably well in most prediction problems and can be used as a benchmark technique. Then, each individual model makes its own prediction estimating probabilities for each class. The final prediction of stacking is computed using multiple-linear regression as a Meta classifier.

The detailed configurations of each individual model are as follows: DTs with C4.5 search algorithm, ANNs are RBF neural network with radial basis functions as activation functions, SVMs use John Platt's sequential minimal optimization algorithm with logistic to the outputs for proper probability estimates.

\subsection{Development the Tool as a Web-Based Application}

Web-based are a common method used for achieving interoperability. The main attractions are that web-based systems are both platform and language 
independent, and there exist standard protocols to facilitate the communication. A Web Services based approach generally involves setting up a web server and some proxy classes in each framework to communicate with this server. Communication is generally achieved through XML-based. .NET Framework and makes it possible both to develop .NET applications in Java, and to use existing Java API's and libraries in applications written in any .NET language. Fig. 2 shows the webbased Intelligent Data Analysis tool that centralizes to support prognosis task. Users can select the desired fields for building classifiers. The designed tool facilitates different types of users to access functionalities in Weka without the need to learn new data mining software.

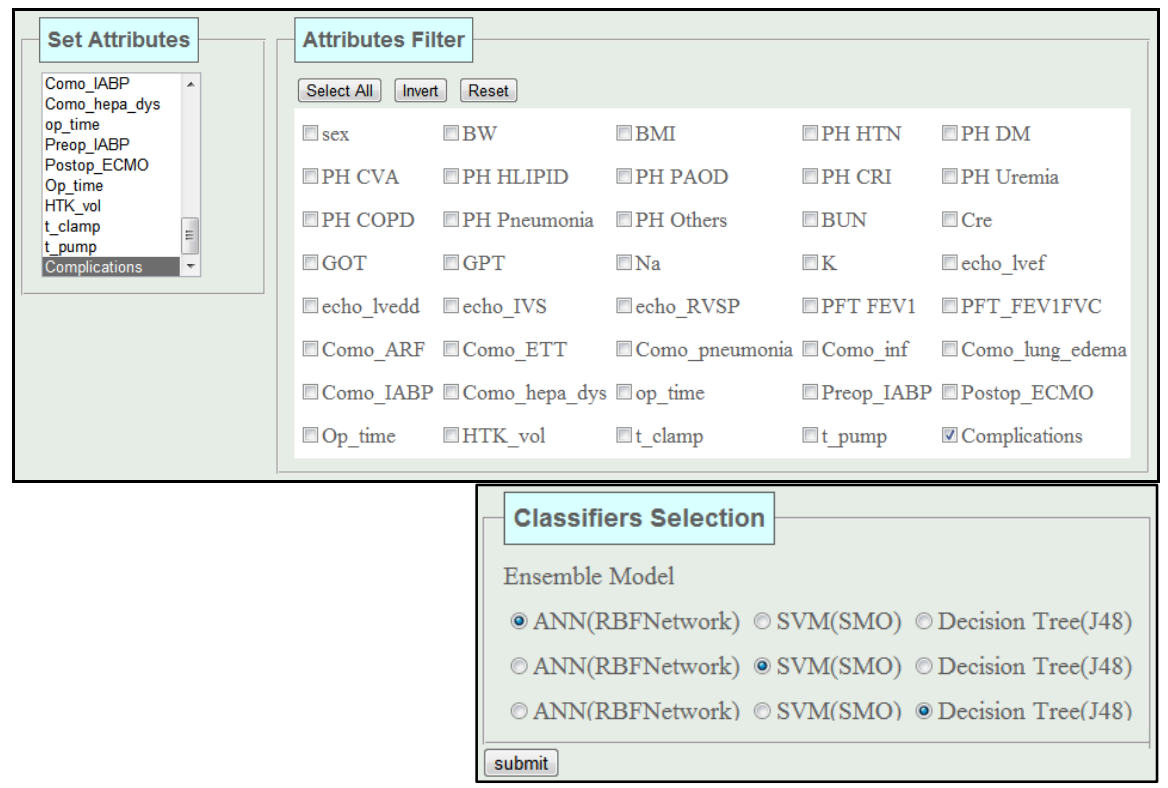

Fig. 2 Attributes filter and model selection

In this study, we intended to develop custom-designed medical data analysis components and schemata, which access the data mining suite's components through Java programming. The following functions were embedded : (1) Basic statistical functions for primary inspection of the data; (2) Visualize data exploratory functions for the interactive of interesting patterns in datasets; (3) Data sources connective and data preprocessing functions for retrieving medical data from databases, sample selection; (4) Unsupervised and supervised data analysis functions for the intelligent data analysis, includes statistical and machine learning algorithms, ex: regression analysis, clustering techniques, association rules, decision trees and support vector machines; (5) Model evaluation functions for the evaluation of built models' reliability and validity, ex: accuracy, sensitivity, specificity, ROC curves and lift chart. 
The model selection scheme is a mixture of bagging and cross-validation (CVbagging) that aims to improve the classification by combining models trained on randomly generated subsets of the entire training set. We first applied a cross validation scheme for model selection on each subset; subsequently, for the sake of simplicity and so as not to run into over-fitting problems, we combined the selected models in a uniform weights approach to reach a final decision. The concept of uniform weights voting is both appealing and simple to implement; it can generally be applied to any type of classifier without relying on specific interpretations of the output. In order to validate models, we used random subsets as crossvalidation folds for reasons of simplicity. In k-fold cross validation, the dataset is partitioned into k subsets. Of these k subsets, a single subset is retained as the validation dataset, and the remaining $\mathrm{k}-1$ subsets are used for training datasets. The cross validation process is then repeated $\mathrm{k}$ times with each of the $\mathrm{k}$ subsets used only once as the validation data. The $\mathrm{k}$ results from the folds can then be averaged to produce a single estimation. In cases where specific inside medical knowledge is not available, such a cross validation method can be used to select a classification method empirically, because it seems to be obvious that no classification method is uniformly superior. We trained several times for individual classifiers and select the best performance one as the final model. The rationale is if we trained each model with different initial conditions, we can find leverage performance for the final model. The result, a heterogeneous ensemble, allows classification methods to be used more effectively.

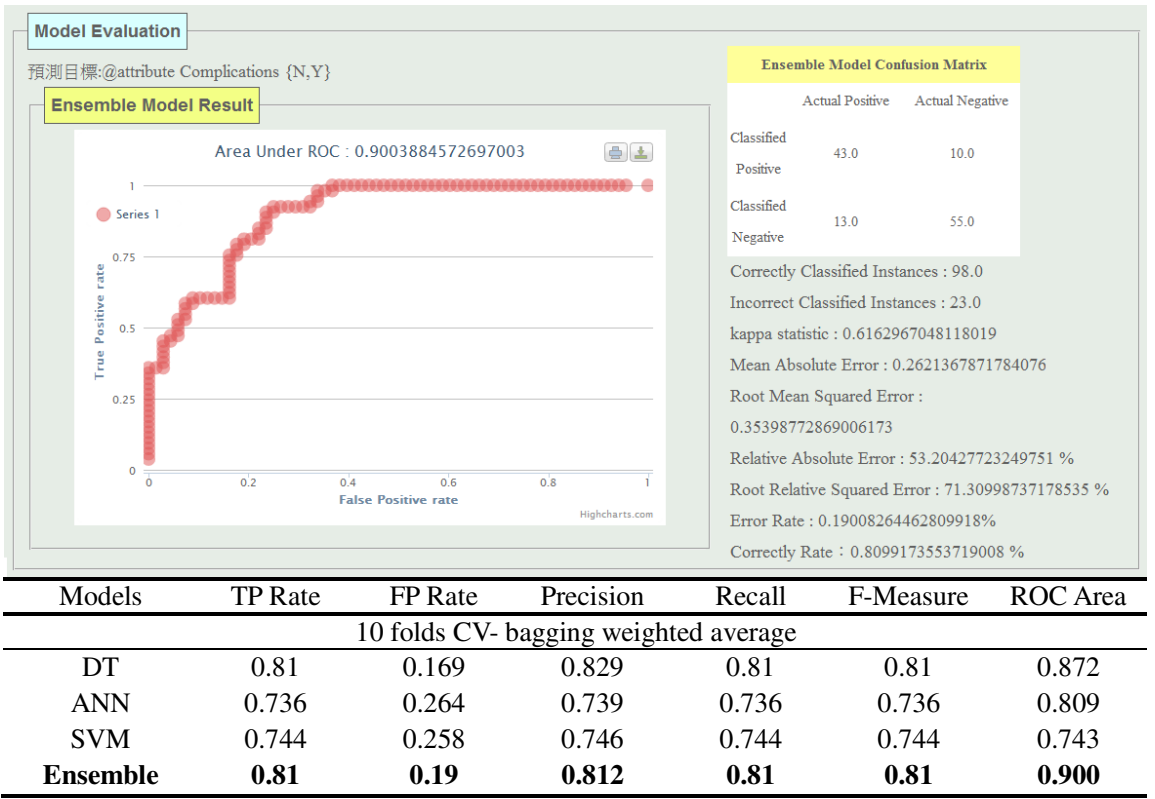

Fig. 3 The results of the prediction 
To show the performance of ensemble model, we used visualization tool, called Receiver operating characteristic (ROC) and Confusion Matrix, which is typically used for model evaluation in the field of artificial intelligence. The area under the ROC Curve is based on a non-parametric statistical sign test, the purpose of which is to estimate the probability of survival for each of a pair of patients. In this study, the area under the ROC was assessed through stratified 10-fold CV-bagging. Each row of the Confusion matrix represents the instances in a predicted class, while each column represents the instances in an actual class. The detailed accuracy of individual models and of the ensemble model is shown in Figure 3.

\section{Results and Discussion}

In open repair, these patients are likely to have a relatively high postoperative morbidity rate with complications, and will highly influence longer-term postoperative out-comes. It is essential to create reliable and satisfactory risk prediction models for postoperative morbidity as an aid to clinical decisionmaking. Although several risk prediction systems have been proposed for patients undergoing open aneurysm repair, they basically rely on traditional statistical methods. We have proposed an ensemble model to predict postoperative morbidity after Open Repair and support clinical decision-making. The proposed ensemble model is constructed by Decision Tree, Artificial Neural Network and Support Vector Machine were used to augment the ensemble model and design a Web-Based Application, showing moderate performance.

The experimental result shows our designed Intelligent Analysis Tool can predict and classify the evolution of patients and facilitates different types of users to access functionalities in Weka, the tool can install and access by any client in the network. The proposed ensemble model predicts postoperative morbidity with relatively satisfactory accuracy, even when data is missing and/or sparse, showing its usefulness in support of clinical decision-making. The supplementary nature of multi-models distinguishes the proposed model from existing risk scoring systems that are based on conventional statistical methods and from various machine learning models. To summarize, the advantage of using the proposed ensemble model is that it can provide surgeons with practical, relatively accurate aid in their daily diagnostic tasks.

\section{References}

1. Bohm, N., Wales, L., Dunckley, M., Morgan, R., Loftus, I., Thompson, M.: Objective risk-scoring systems for repair of abdominal aortic aneurysms: applicability in Endovascular repair. European Journal of Vascular and Endovascular Surgery 36, 172-177 (2008)

2. Stijn, C.W., Wouters, L.N., Freek, W.A.V., Rene, M.H.J.B.: Preoperative prediction of early mortality and morbidity in coronary bypass surgery. Cardiovascular Surgery 10, 500-505 (2002) 
3. Barnes, M., Boult, M., Maddern, G., Fitridge, R.: A model to predict outcomes for Endovascular aneurysm repair using preoperative variables. European Journal of Vascular and Endovascular Surgery 35, 571-579 (2008)

4. Delen, D., Sharda, R., Kumar, P.: Movie forecast Guru: A Web-based DSS for Hollywood managers. Decision Support Systems 43(4), 1151-1570 (2007)

5. Murphy, C.K.: Identifying diagnostic errors with induced decision trees. Medical Decision Making 21(5), 368-375 (2001)

6. Eom, J.-H., Kim, S.-C., Zhang, B.-T.: AptaCDSS-E: A classifier ensemble-based clinical decision support system for cardiovascular disease level prediction. Expert Systems with Applications 34, 2465-2479 (2008)

7. Polat, K., Güne, S.: Breast cancer diagnosis using least square support vector machine. Digital Signal Processing 17, 694-701 (2007)

8. Babaoğlu, I., Fındık, O., Bayrak, M.: Effects of principle component analysis on assessment of coronary artery diseases using support vector machine. Expert Systems with Applications 37(3), 2182-2185 (2010)

9. Choi, S.: Detection of valvular heart disorders using wavelet packet decomposition and support vector machine. Expert Systems with Applications 35, 1679-1687 (2008)

10. Witten, I.H., Frank, E., Hall, M.A.: Data Mining: Practical machine learning tools and techniques, 3rd edn. Morgan Kaufmann, San Francisco (2011) 